\title{
Effects of Media with Violent Content on College Students' Aggressive Reaction
}

\author{
Pedro Esteban Negrete Narvaez, Robert J. F. Elsner \\ Erskine College, Due West, USA
}

\begin{abstract}
The effect of media violence on aggression levels was explored and related to increases in aggression levels based on personality traits. In this study, 78 undergraduate students were divided into a control group (violent video) and an experimental group (non-violent video) using a $2 \times 2$ factorial design (two video clips, two aggression inventories). The participants completed a pre-test Buss-Perry Aggression Questionnaire and the Mini-IPIP prior to being exposed to one of the two video clips. Afterwards the video exposure, the participants completed a post-test Buss-Perry Aggression Questionnaire. Analysis of the participants' data $(n=78)$, showed verbal aggression and conscientiousness approached significance, $F(1,77)=3.750, p<0.057$, as did verbal aggression and agreeableness, $F(1,77)=3.796, p<0.055$. A significant difference was found in anger with neuroticism, $F(1,77)=7.577$, $p<0.008$. Overall anger and aggression levels pre- and post-exposure were closely related, indicating that some were already angry and aggressive $t(77)=2.3769, p<0.020$. Furthermore, control and the experimental groups only approximated significance in physical aggression $F(1,77)=3.832, p<0.054$. Contrary to previous studies, exposure to violence did not increase aggressive behavior as hypothesized; however, these findings showed that violence in media had an effect on the factor of anger and a difference between both groups in physical aggression. In addition, exposure to violence had an effect on specific personality traits of participants, such as agreeableness, conscientiousness, and neuroticism.
\end{abstract}

Keywords: aggression, personality, violence exposure, aggressive behavior, media exposure

Aggression and violence are topics mentioned often as an influence on many different areas of one's life. Violence can be found on TV, in cartoons, or in live shows. TV violence exposure is considered to be a contributor that increases aggressive behavior (Pinto da Mota Matos, Alves Ferreira, \& Haase, 2012). Just as the Bobo doll experiment showed (Bandura, Ross, \& Ross, 1961), children learn aggression through observation. Mussen and Rutherford (1961) showed that children who are exposed to violent cartoons had a violent response afterwards, whereas, the children who were not exposed to the same content had a non-violent response. Pinto da Mota Matos et al. (2012) analyzed violence on television, in which the results showed that the relationship between TV violence, physical aggression, and verbal aggression are mediated by enjoyment of TV violence (Pinto da Mota Matos et al., 2012). Shafron and Karno (2013) also mention that violent music has an effect on the listener arousing their aggression tendencies.

Video games are another form of media which contain violence that increases aggressive behavior in their

Pedro Esteban Negrete Narvaez, Ph.D., Dept. of Psychology, Erskine College.

Robert J. F. Elsner, Dept. of Psychology, Erskine College. 
players. A study showed that violent video games affect the players exposed to this medium by increasing their physical aggression when high in trait anger (Engelhardt, Bartholow, \& Saults, 2011). In addition, Przybylski, Deci, Rigby, and Ryan (2014), found "that competence-impeding play led to higher levels of aggressive feelings, easier access to aggressive thoughts, and a greater likelihood of enacting aggressive behavior" (p. 441).

Sports are another medium in which violence is found and even permitted in some cases. Kerr (2009) mentions four types of violent behavior that are applied in sports: Anger violence (retributive), thrill violence (provocative), power violence (procedural), and play violence (recreational). Soccer is not a violent sport, but players can play aggressively and have physical contact with other players (play violence). But even here, violence can go further with other violent acts in the game, like fouls and fights. These acts include intentionally attacking another player and with the purpose of hurting them, making usage of the thrill, anger, and power violence as violent behavior (Kerr, 2009).

The internet is another source where violence is pervasive, including negative content related to aggression in social media, such as the cyber bulling on Facebook, "verbal" fights on Twitter, or in pictures and videos posted on Instagram. YouTube has been infested with videos of school fights, compilations of fights in sports, law enforcers vs. civilians, and other common acts of violence. This violence in media has been researched previously with results yielding the increase of aggressive behavior from audiences exposed to this content in movies, videos, video games, TV and more (Krahé \& Busching, 2015). Webster et al. (2014) state that "specifically, trait aggression describes individual differences in thoughts (e.g., hostility), emotions (e.g., anger), and behavior (e.g., verbal and Physical Aggression) that are intended to harm another person" (p. 121).

"Self-determination theory researchers have argued that people are more prone to aggression when any of these three basic needs - (competence, autonomy, and relatedness) - is thwarted either proximally, by situational threats or deprivations, or distally, by chronic developmental conditions" (Przybylski et al., 2014, p. 441). Aggression can be divided into proactive and reactive: proactive being the creation or control of a situation that will cause a reaction on someone else, reactive being the aggression type of expression of anger as a response to a stimulus affecting the feeling of anger (Palme \& Thakordas, 2005).

The aggressive reactions, either an arousal or dissuade, were tested in terms of personality traits. For example, if a person watches a video of a sport fight or unfair play, it would be tested to see how the participant reacts toward the video; before and after while linking this reaction to personality traits. Personality traits are different among individuals, but, simultaneously, the traits are considered to remain stable within the pass of time (Karimizadeh, Mahnam, Yazdchi, \& Besharat, 2015). Therefore, personality played an important role in this study since it was considered a factor that might have a relationship with the dimensions of aggression. It can be considered that aggression levels could increase or decrease (be affected) by certain factors of personality. Low scores on agreeableness and conscientiousness and high scores on neuroticism have been related to aggression, adding that these ones could reinforce aggressive behavior (Hosie, Gilbert, Simpson, \& Daffern, 2014).

The purpose of the present study was to explore relationships between violent content and aggressive reaction after exposure to violent content as mediated by personality. It was hypothesized that a person would react toward the violent video by having an aggressive reaction, while the person exposed to the non-violent video should not have an aggressive reaction. 


\section{Methods}

\section{Participants}

Seventy-eight undergraduate students from a small, rural, private, liberal arts, church affiliated college, located in the southeast region of the United States were recruited. Participants identified as 47 females and 31 males (17-24 years old); Caucasian (61.53\%), African American (25.64\%), Hispanic (6.41\%), Asian (3.84\%), and Other $(2.56 \%)$. Participants provided informed consent before the experiment took place. All American Psychological Association and Helsinki Declaration Ethical standards were adhered to in this project, which had already been approved by the Institutional Review Board.

\section{Research Design}

The participant's personality was examined in the study in order to be analyzed with the purpose of finding a relationship and difference between the aggressiveness of the sample and their personality. A $2 \times 2$ factorial design was used for this research. The 2 videos were the independent variables, which were hypothesized to have an effect on the person's aggressive reaction. The aggressiveness pre- and post-exposure were measured for the experiment, acting as the dependent variables. The Personality traits of each participant were measured as well. In this case, personality played the role of a standard variable, a factor that was added to find a link or distinction to the levels of aggression.

\section{Materials}

The Mini-IPIP (Donnellan, Oswald, Baird, \& Lucas, 2006), was used to assess the personality of the participants. The Mini-IPIP is 20-item short version of the 50-item IPIP, Five-Factor Model measure (Goldberg, 1999). The Mini-IPIP measures extraversion, agreeableness, conscientiousness, neuroticism, and intellect/imagination, a variation of the Big Five Inventory (Webster et al., 2104). The items in the inventory are rated on a 1 to 5 Likert scale, 1 being "Very inaccurate" and 5 being "Very accurate".

The Buss-Perry Aggression Questionnaire (BPAQ) (Buss \& Perry, 1992) assessed the participants' aggression in several different dimensions. The BPAQ is a 29 item questionnaire, in which each item is ranked on a 5 point Likert scale: 1 being "Extremely uncharacteristic" to 5 being "Extremely characteristic". This questionnaire analyzes four dimensions of aggression: physical aggression, verbal aggression, anger, and hostility. Because the BPAQ was used to measure the dimensions of aggression twice (pre- and post-exposure to media), administration was changed to reduce exposure bias. Statements from the BPAQ were recorded as an audio clip with a gap of 3 seconds in between each of the statements, so that the participants would not be able see the statements from the pre-exposure measurements.

The video used for the experimental group was a compilation of fights in rugby with a length of approximately 5 minutes. This sport was chosen because it is not a common or well-known sport on the campus. The second video, used for the control group, was a fun and entertaining video from Walt Disney Animation Studios, "Paperman".

\section{Procedure}

Participants were randomly assigned to a computer in the Psychology Department iMac lab where participants took the Buss-Perry Aggression Questionnaire in the audio clip version as the baseline of the aggressiveness levels pre-exposure. Participants listened to each of the statements using headphones, answering on paper. Then, the participants proceeded to fill out the Mini-IPIP, the demographic form, and then proceeded 
to watch one of the videos. When the video clip ended, the participants completed the Buss-Perry Aggression Questionnaire again in order to have a measurement of their reaction after the media exposure. This time the questionnaire contained the statements in paper form.

\section{Results}

The data collected was entered into Microsoft Excel and validated using a 1:5 random pull sequence of $20 \%$ of the data, then analyzed using SPSS (v. 23, IBM Corp, Armonk, NY, USA). Multivariate analysis on physical aggression between the experimental group and the control group only approached significance, $F(1,77)=3.832, p<0.054$. Univariate ANOVA showed two approximations of significance; verbal aggression and conscientiousness with $F(1,77)=3.750, p<0.057$ and verbal aggression and agreeableness with $F(1,77)=3.796, p<0.055$. This one also showed a significant value between anger and neuroticism with a value of $F(1,77)=7.537, p<0.008$. Overall changes in hostility were associated only with the personality aspect of conscientiousness, $F(11,66)=3.057, p<0.002$. Correlations showed a relationship between verbal aggression and conscientiousness, pre-exposure $r(78)=0.299, p<0.008$, post-exposure $r(78)=0.310$, $p<0.006$. It also showed a relationship between anger and neuroticism, pre-exposure $r(78)=0.420, p<0.001$, post-exposure $r(78)=0.458, p<0.001$.

\section{Discussion}

It was expected that, like in Mussen's and Rutherford (1961), exposure to media containing violent content would cause an effect on the participants; but even though the results of the paired samples $t$-test showed significance on the dimension of anger, the value decreased in the sample based on the means. Pre-exposure mean $M=13.40, S D=3.085$ and post-exposure $M=12.85, S D=3.094$. Nevertheless, the paired samples results were analyzed containing both the experimental and the control group data.

Violence in media did not have an effect on increasing aggressive behavior, however, the multivariate analysis showed that there was a difference in physical aggression between the experimental group and the control group. According to Engelhardt, Bartholow, and Saults (2011), the participants high in trait anger were the most aggressive in physical aggression, but only if they first played a violent video game. The differentiation between the two groups in the post-exposure scores in the current study support these claims. This might be because people exposed to the violent content had an effect on physical aggression.

For the personality traits and the dimensions of aggression, there was a difference on verbal aggression with conscientiousness, verbal aggression with agreeableness, and anger with neuroticism. This shows that a pattern was found in the data regarding those personality traits and the dimensions of aggression. Consistent with the literature regarding personality traits, "trait aggression often relates positively with neuroticism - and negatively with agreeableness, openness, and conscientiousness" (Webster et al., 2014, p. 121), similar to the findings of the current study yielding a link between the personality traits and the dimensions of aggression.

At the same time, correlations showed that there was a relationship or connection between verbal aggression with conscientiousness, and anger with neuroticism. According to the findings of previous research by Hosie et al. (2014), "aggression was related to low agreeableness and low conscientiousness. The relationship between GAM (General Aggression Model) specified constructs with the FFM (Five Factor Model) constructs revealed that Agreeableness, Conscientiousness, and Neuroticism were correlated with STAXI Trait 
Anger" (p. 194). The findings in the current study provide a general indication of the strong relation that certain traits have with the dimensions of aggression.

Results of the univariate ANOVA and the correlation indicate the same personality traits and the type of aggression as well as the same personality traits mentioned by the literature. It is suggested that further research be conducted to explore the effects between the personality traits and the dimensions of aggression to see if there is a specific reason why the results yielded agreeableness, conscientiousness, and neuroticism as significant.

Pinto da Mota Matos (2012) had suggested that for future studies, it might be considered that the increase of aggressive reaction might be affected by the enjoyment of the violent content. Even though the current study experimented with video clips, Oliver et al. (2015), mention that the enjoyment of certain video games comes from the game characteristics and satisfaction of needs which makes them fun. Participants for the current study had been recruited from a small church-affiliated school, and previous studies at the institution had indicated an overall low level of aggressiveness. In the current study, it was observed that certain participants laughed during the exposure to the violent video and a few even asked the researcher if the videos could be more violent and perhaps bloody next time, implying the enjoyment of the content showed. In a larger sample size, the effects of personality on violence and aggression would likely be more pronounced.

\section{References}

Bandura, A., Ross, D., \& Ross, S. A. (1961). Transmission of aggression through imitation of aggressive models. The Journal of Abnormal and Social Psychology, 63, 575-582. doi:10.1037/h0045925

Buss, A. H., \& Perry, M. P. (1992). The aggression questionnaire. Journal of Personality and Social Psychology, 63, $452-459$.

Donnellan, M. B., Oswald, F. L., Baird, B. M., \& Lucas, R. E. (2006). The mini-IPIP scales: Tiny-yet-effective measures of the Big Five factors of personality. Psychological Assessment, 18, 192-203.

Engelhardt, C. R., Bartholow, B. D., \& Saults, J. S. (2011). Violent and nonviolent video games differentially affect physical aggression for individuals high vs. low in dispositional anger. Aggressive Behavior, 37, 539-546.

Goldberg, L. R. (1999). A broad-bandwidth, public domain, personality inventory measuring the lower-level facets of several five-factor models. In I. Mervielde, I. Deary, F. De Fruyt, \& F. Ostendorf (Eds.), Personality psychology in Europe (Vol. 7, pp. 7-28). Tilburg, The Netherlands: Tilburg University Press.

Hosie, J., Gilbert, F., Simpson, K., \& Daffern, M. (2014). An examination of the relationship between personality and aggression using the general aggression and five factor models. Aggressive Behavior, 40, 189-196.

John, O. P., \& Srivastava, S. (1999). The Big-Five trait taxonomy: History, measurement, and theoretical perspectives. In L. A. Pervin, \& O. P. John (Eds.), Handbook of personality: Theory and research (2nd ed., pp. 102-138). New York: Guildford Press.

Kahrs, J. (Director), Reed, K. (Producer), \& Chiang, C., \& Hoyer, K. (Writers). (2012). Paperman (Motion picture). United States: Walt Disney Animation Studios.

Karimizadeh, A., Mahnam, A., Yazdchi, M. R., \& Besharat, M. A. (2015). Individual differences in personality traits: Perfectionism and the brain structure. Journal of Psychophysiology, 29, 107-111. doi:10.1027/0269-8803/a000141

Kerr, J. H. (2009). Analysis of recent incidents of on-field violence in sport: Legal decisions and additional considerations from psychology. Aggressive Behavior, 35, 41-48.

Krahé, B., \& Busching, R. (2015). Breaking the vicious cycle of media violence use and aggression: A test of intervention effects over 30 months. Psychology of Violence, 5, 217-226. doi:10.1037/a0036627

Mussen, P., \& Rutherford, E. (1961). Effects of aggressive cartoons on children's aggressive play. The Journal of Abnormal and Social Psychology, 62, 461-464. doi:10.1037/h0045672

(Napoleon PSVita). (2014, October 29). Rugby Fights of 2014-Brawl and Fights HD (Video File). Retrieved from https://www.youtube.com/watch?v=DIB48HnEB2I

Oliver, M. B., Bowman, N. D., Woolley, J. K., Rogers, R., Sherrick, B. I., \& Chung, M. (2015). Video games as meaningful entertainment experiences. Psychology of Popular Media Culture, doi:10.1037/ppm0000066 
Palme, E. J., \& Thakordas, V. (2005). Relationship between bullying and scores on the buss-Perry aggression questionnaire among imprisoned male offenders. Aggressive Behavior, 31, 56-66. doi:10.1002/ab.20072

Pinto da Mota Matos, A., Alves Ferreira, J. G., \& Haase, R. F. (2012). Television and aggression: A test of a mediated model with a sample of Portuguese students. The Journal of Social Psychology, 152, 75-91.

Przybylski, A. K., Deci, E. L., Rigby, C. S., \& Ryan, R. M. (2014). Competence-impeding electronic games and players' aggressive feelings, thoughts, and behaviors. Journal of Personality and Social Psychology, 106, 441-457. doi: $10.1037 / \mathrm{a} 0034820$

Shafron, G. R., \& Karno, M. P. (2013). Heavy metal music and emotional dysphoria among listeners. Psychology of Popular Media Culture, 2, 74-85. doi:10.1037/a0031722

Swami, V., Pietschnig, J., Bertl, B., Nader, I. W., Stieger, S., \& Voracek, M. (2012). Personality differences between tattooed and nontattooed individuals. Psychological Reports, 111, 97-106. doi:10.2466/09.07.21.PR0.111.4.97-106

Webster, G. D., DeWall, C. N., Pond, R. S., Deckman, T., Jonason, P. K., Le, B. M., \& ... Bator, R. J. (2014). The brief aggression questionnaire: Psychometric and behavioral evidence for an efficient measure of trait aggression. Aggressive Behavior, 40, 120-139. 